\title{
Engagiertes Fragen: Hubert Fichtes Milieu-Studien
}

\author{
Dorothea Walzer \\ Ruhr-Universität Bochum, Germanistisches Institut, \\ Universitätsstr. 150, 4478o Bochum \\ dorothea.walzer@rub.de
}

Hubert Fichtes gesamtes Werk ist von dem Insistieren der Frage gekennzeichnet; seine Romane, seine Dramen und Novellen, seine Features und Hörstücke sowie die gemeinsam mit der Fotografin Leonore Mau erarbeiteten Fotofilme basieren auf Interviews, die Fichte über die Jahre in unterschiedlichsten Milieus und Ländern geführt und zu einem riesigen Archiv von Stimmen zusammengestellt hat. Er führt Interviews mit Autoren wie Jean Genet, Salvador Allende oder der Popkultur-Ikone Lil Picard ebenso wie mit Arbeitern aus dem Hamburger Hafen, mit portugiesischen Fischern oder Priestern afroamerikanischer Religionen, mit Strichern und Zuhältern, Gammlern und Hippies.

Von den frühen 196oer Jahren bis zu seinem Tod im Jahre 1986 wird Fichte als ein engagierter Intellektueller auftreten, der mit seiner Interview-Literatur eine Oral History anderer Art praktiziert. Denn das Interview wird von Fichte als „ethnopoetisches" ${ }^{\text {1 }}$ Forschungs- und Darstellungstool gebraucht, um Größen aus Protest- und Subkultur sowie kleinen und delinquenten Leuten eine Stimme zu geben, wobei mit dem Befragten auch der Fragende und die Interview- oder Fragesituation selbst, ihre Bedingungen und Setzungen in den Blick geraten. Fichtes Interview-Literatur erlaubt es, das Fragen selbst zu befragen. Was damit zur Debatte steht, ist die Konstruktion und die Problematisierung von Autor- und Zeugenschaft, mithin ein Punkt, an dem eine der wesentlichen Fragestellungen literarischer Produktion mit einer Problemstellung historiographischer oder dokumentarischer Schreibweisen zusammenfällt. Interpretieren wir das Interview ganz grundlegend als Schauplatz einer sekundären oder doppelten, einer arbeitsteiligen oder schlicht bedingten Autorschaft, so gilt dies nämlich nicht erst für jenen Moment, in dem das Sprechen aufgenommen und womöglich im Prozess der Transkription und der Niederschrift verändert wird; es gilt bereits dort, wo die Gewaltsamkeit der Frage zur Aussage zwingt oder die Frage ein bestimmtes Möglichkeitsspektrum

1 Vgl. Böhme (1992), insbes. S. 29-36; Böhme (1992b), S. 247-275. 
des Antwortens mitcodiert. Wer spricht, wenn der Befehl zu sprechen ausgegeben ist? Inwieweit sprechen die institutionelle Anordnung und die medialen Arrangements, die Modelle und Vorlagen, aber auch die Neugierde oder das Begehren des Fragenden in der Antwort bereits mit? Und auf welche Weise prägen die Aufzeichnungs-, die Transkriptions- und Bearbeitungsprozesse, die das Interview notwendig durchläuft, unser Wissen von dem, was wir zuletzt als primäre Interviewsituation imaginieren? Wie also bezieht sich Fichtes Ethnopoesie auf die Werkzeuge und Verfahren des eigenen Forschungsunternehmens, auf die Produktions- und Reproduktionsbedingungen der Interview-Episteme insgesamt und die ganz konkreten Prä-, Sub- und Kontexte der jeweiligen Befragung?

\section{Versuch über das Interview}

Mit dem Roman Forschungsbericht, der, im Jahre 1980 geschrieben, nach Fichtes Tod im Rahmen seiner 17-bändigen Geschichte der Empfindlichkeit erscheinen wird, legt der Autor einen literarischen Versuch über das Textgenre des Interviews vor, das er als primäres Werkzeug seines ethnopoetischen Schaffens einsetzt. Zusammen mit der Fotografin, seiner Partnerin Leonore Mau macht sich Fichte auf Forschungsreise zu den schwarzen Kariben in Dangriga, um deren Gebräuche und Riten zu befragen, so der Plot des Romans. Das Interview, das der Begegnung mit einem unzugänglichen Gegenüber dienen, dessen Erfahrungen einsammeln und zugänglich machen soll, kann seinem Versprechen jedoch von Beginn an nicht gerecht werden. In Fichtes Forschungsversuch wird ausgerechnet das scheiternde Interview-Gespräch zum insistierenden Motiv und Ausgangspunkt des literarischen Schreibens gemacht. ${ }^{2}$ Wenn die Befragung nur austauschbare Antworten abwirft oder als orgasmische Begeg-

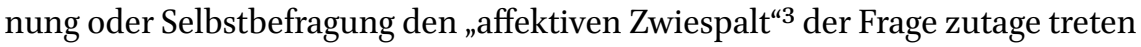
lässt, scheitern Ermittlung und Begegnung, Aufklärung und Erkennen in ein und demselben Zuge. Deutlich wird dabei, dass das Interview in Fichtes Literatur keineswegs als Medium fungiert, das zu kategorisieren, zu beurteilen und die Wahrheit über einen anderen zu ermitteln erlaubt; es ist vielmehr ein Medium, das die Fähigkeit zu unterscheiden und zu beurteilen ganz grundlegend problematisiert. Und das betrifft an erster Stelle den Status des Autorsubjekts

2 Vgl. dazu Bies (2014), S. 148.

3 Hartmut Böhme macht deutlich, daß die „Leidenschaft, die im Fragen wirkt..." auch „[i]m scheinbar sachlich neutralen Einholen von Wissen“ ihre Spuren hinterläßt (Böhme (1992b), S. 266). 
und den Status der Aussage, kurz: die Frage, wer auf welche Weise zum Zeugen werden kann. Denn die Frage qui parle? - wer spricht hier eigentlich, der Befragte, der Fragende oder gar das Frage-Begehren ${ }^{4}$ des letzteren? - ist in Fichtes Interviews kaum zu klären.

Da zu Fichtes Forschungsbericht bereits viel geschrieben wurde, möchte ich mich im Folgenden mit einem anderen Interview-Text Fichtes, genauer mit einem Ensemble von Interviews befassen, das sich gewissermaßen als praktische Übung über das Genre des Interviews und die möglichen Formen seiner Bearbeitung lesen lässt. Unter Fichtes Interviewpartnern findet sich einer, den Fichte innerhalb von dreizehn Jahren mehrere Male befragen und mit dem er im Laufe dieser Zeit eine enge freundschaftliche, von Streit und Versöhnung, Identifikation und Begehren gekennzeichnete Beziehung aufbauen wird. Es handelt sich um den Zuhälter Wolli, Besitzer einer Etage des staatlich subventionierten Bordells mit dem Namen Palais d'Amour, einen kleinen Mann aus dem Rotlichtmilieu Hamburgs, der doch unbestreitbar als Kiezgröße bezeichnet werden kann; um einen schnoddrigen Aussteiger und Slacker, der doch ein Kenner der großen Literatur und der Kunst, ja sogar Kunstsammler ist. Nicht nur die viermalige Wiederaufnahme des Interviewgesprächs mit diesem Gesprächspartner, sondern auch dessen Verarbeitung in unterschiedlichsten Medien und Genres - als Interviewroman, als Hörspielinszenierung und Gesprächsdokumentation - deutet an, dass wir es hier mit einem Projekt zu tun haben, das sich durch seine Vorläufigkeit und seinen experimentellen Charakter auszeichnet. Und tatsächlich zeigt sich bei genauerer Untersuchung, dass dieses Fortsetzungsprojekt im selben Moment, in dem es Wissen über die gelebten Leben der befragten Individuen akkumuliert, eine Arbeit an den Grundlagen, den Instrumenten und Verarbeitungsformen des im Interview gewonnenen Wissens ist.

Es entstehen Interviewtexte, die dokumentarisch und fiktional, authentisch und inszeniert zugleich sind, die die Poetizität der im Interview gewonnenen Aussage ganz offensiv ausstellen und damit deutlich machen, dass Wissen, um erscheinen zu können, auf Darstellung nicht verzichten kann. Vor allem aber erkunden sie den Status der Autor- und Zeugenschaft im Interview, wenn sie das Interview als kontextsensibles Verfahren in Szene setzen, als ein Verfahren also, das die Entwicklung des Textes vorantreibt, indem es ihn auf seine unterschiedlichen Kontexte hin öffnet. Letzteres ist - wie ich an dieser Stelle vorwegschicken möchte - gerade deswegen so interessant, weil es die Vollendung

4 In einem wegweisenden Artikel über Fichtes „Reise-Begehren“ hat Dirck Linck auf den Nexus von Wissen und schwulem Begehren aufmerksam gemacht, die Frage als Werkzeug eines solchen Begehrens aber nicht weiter in seine Überlegungen einbezogen (Linck (1995), S. 23-54). 
des Erkenntnis- und Urteilsprozesses, die erschöpfende Kategorisierung und Beurteilung des Portraitierten auf verfahrenstechnischer und formaler Ebene ganz grundsätzlich unterbindet. Durch die Eintragung eines textkonstitutiven Außen - der Prä- und Sub-, der Produktions- und der Rezeptionstexte - in den Text selbst werden permanent Akzentverschiebungen erzeugt, womit ein abschließendes Ergebnis der gemeinsamen Denk- und Schreibbemühungen fortwährend aufgeschoben bleibt. Eine Akzentverschiebung im Sprechen verweist nämlich, wie Samuel Weber es in einem Vorwort zur Sprachphilosophie des marxistischen Sprachtheoretikers Valentin N. Vološinov formuliert hat, „nicht mehr auf ein Subjekt - auch nicht auf ein schreibendes, erzählendes, wiederholendes - das sie ganz zu verantworten hätte, sondern sie verweist auf den Text, der kein letztes Wort mehr kennt, und auch kein erstes, kein Autorenwort und auch kein fremdes “. ${ }^{5}$ Das Scheitern wird insofern als Aufschub jedes möglichen Ergebnisses umgedeutet; da es ein nicht endenwollendes Gespräch ins Leben ruft, bürgt es auch hier für den Erfolg der Frage-Methode.

\section{Wolli Indienfahrer}

Nach langen Vorgesprächen und Erkundungen im Milieu des Hamburger Kiezes führt Fichte 1969 das erste einer Reihe von Interviews mit dem damals erst 37-jährigen Wolli. Es folgen zwei weitere Interviews - eines entsteht in demselben Jahr, das andere ganze acht Jahre später. Von Fichte erstmals 1978 als Trilogie unter den Titeln "Wolli I“, „Wolli II" und „Wolli III" in dem Interview-Band Wolli Indienfahrer, roman publiziert - ergänzt durch eine Reihe von Interviews mit Strichern und Prostituierten, Angestellten des Palais d'Amour, die ebenfalls 1969 geführt wurden -, blickt der Roman auf eine bezeichnende Publikationsgeschichte zurück. Denn während Fichte die Gesprächsprotokolle, also die Verschriftung der mündlich geführten Interviewgespräche mit Ulli und Gunda, Jonny und Sandra, bereits 1972 in dem Band Interviews aus dem Palais d'Amour etc. ${ }^{6}$ publiziert hatte, stellt sich Wolli zu diesem Zeitpunkt im Streit gegen eine Veröffentlichung seiner Interviews. Doch das „etc.“ zeigt an, dass das letzte Wort noch nicht gesprochen ist. Und so kann der Band sechs Jahre später in erweiterter Form erscheinen: Wollis Interviews sind nun zentraler Bestandteil des Bandes; dies jedoch in kommentierter Form. So hatte Fichte seinem Gesprächspartner die Abschriften der Tonbandmitschnitte schließlich

5 Weber (1975), S. 45 .

6 Fichte (1972). 
zur Durchsicht vorgelegt und dessen Kommentare in den Interview-Text eingetragen.

Was entsteht, ist ein komplexes Bild der Lebens- und Arbeitsbedingungen, der kulturellen Vorlieben, der sexuellen Präferenzen und Wünsche eines Zuhälters und seiner Beschäftigten, ein facettenreiches Panorama des Kiez-Milieus. ${ }^{7}$ Ungewöhnlich schon deswegen, weil es das Milieu der Befragten ebenso abbildet wie die Umstände seiner Befragung. Fichtes Oral History beschränkt sich also keineswegs darauf, zwielichtige subproletarische Gestalten zu Wort kommen zu lassen, sie erzählen zu lassen, was sie sich selbst und anderen zu erzählen pflegen - eine Tendenz, die das klischeebeladene Kiez-Milieu in besonderem Maße nahelegt, wie Fichte den interviewten Wolli selbst erläutern lässt. ${ }^{8}$ Stattdessen gelingt es ihm, seine Milieu-Studie als Chronik der Neigungen, der Begierden und Sehnsüchte, aber auch der Ängste und Ressentiments zu entwerfen, welche das Begehren des anderen ebenso in den Blick rückt wie das Frage-Begehren des Interviewleiters selbst. ${ }^{9}$

Um den nicht erzählten und nicht erzählbaren Geschichten näher zu kommen, erarbeitet Fichte einen Handapparat von Fragetechniken, der sich über die Jahre zwar etwas verändert, dessen Konturen sich aber dennoch sehr klar durch Interviews mit unterschiedlichsten Protagonisten ziehen. Die Entscheidung, nachzufragen statt sich mit Aussagen zufriedenzugeben, sie als verhandelbare und eskalierbare Artikulationsformen zu verstehen, die gerade in ihren widersprüchlichen und spekulativen Momenten an die schwer zugänglichen und nicht-intelligiblen Subtexte des Alltäglichen sowie des Forschungsunternehmens selbst rühren, scheint sein Vorgehen grundlegend zu bestimmen. Dabei sind es - das führt uns zum Kern von Fichtes Fragen immer wieder die Fragen nach den Umständen, die seine Gesprächspartner an jene Umschlagpunkte heranführen, an denen gesagt wird, was eigentlich verschwiegen werden sollte. ${ }^{10}$ Die minutiöse Erkundung der Lebensumstände

7 Und tatsächlich zeigt sich in dieser Sammlung, dass nicht erst die späten Romane $E x$ plosion oder Das Haus der Mina in São Luiz de Maranhão, die im Rahmen von Fichtes Geschichte der Empfindlichkeit entstehen, sondern bereits seine frühen Interview-Portraits literarische Collagen aus „tausend widersprüchlichen Fitzeln“ sind. Vgl. Seifert-Waibel (2005).

$8 \quad$ Fichte (1983), S. 78.

9 Eine Chronik der Gefühle entwirft Fichte mit seinen Interviewbänden also schon lange bevor er 1974 nach dem Vorbild von Proust eine Geschichte der Empfindlichkeit zu schreiben beginnt.

10 Mit Blick auf die Interviewführung des Regisseurs und Autors Alexander Kluge hat Joseph Vogl die sogenannten „Umstandsfragen“ in den Blick gerückt, „die sich auf das Akzidens, auf das Mannigfaltige und schlicht auf das ,Ereignis" beziehen“ (Vogl (2007), S. 124). 
seines Gegenübers gehört dazu ebenso wie Fragen, die sich auf die Umstände der Interviewsituation richten. Milieu-Studie ist diese Studie von daher im doppelten Sinne des Wortes.

Dass Fichte sich plakativ auf die impliziten Voraussetzungen des Interviews auf vorherige Begegnungen, Recherchen und Vorgespräche, aber auch auf die Beziehung der Gesprächspartner und den rahmenden Kontext - bezieht, wird in den Eingangs- und Ausgangssequenzen seiner Interviews besonders deutlich. ${ }^{11}$ So etwa, wenn er zu Beginn des zweiten Interviews eine fast szenische Beschreibung der Interviewsituation entwirft, um das Gespräch zu eröffnen:

Fichte: Was hast du da aufgelegt, Wolli?

Wolli: Miles Davis.

Fichte: Du, wir sitzen hier in deiner Wohnung, an der Wand sind Bilder von Generalize, Klaus Stoldt, Buffet, Hausner, Magritte, Klopottan und Schindehütte.

Wolli: Katinka Niederstrasser. Da hab ich übrigens einen ganz irren Katalog hier von dieser Sammlung, wo der Artmann die Gedichte zu gemacht hat, was er uns damals vorgelesen hat, zum Teil mit den Texten, die ganze Mappe kannst du noch kaufen, ich glaube so was um $500 .{ }^{12}$

Nachdem die Befragungssituation geschildert ist, die gleichsam ein erstes Licht auf das heimische Milieu und das darin angezeigte "kulturelle Kapital ${ }^{43}$ seines Gesprächspartners Wolli wirft, geht Fichte kurz auf das Verfahren der Befragung ein, um dann sehr plötzlich einen Szenenwechsel zu vollziehen: auf ein vorhergehendes Ereignis Bezug nehmend, kommt er auf einen Bereich von Wollis Privatleben zu sprechen, den sein Gegenüber, wie sich später herausstellen wird, lieber nicht diskutieren möchte:

Fichte: Ich komme nun hier mit einem Zettel, mit lauter Fragen, weil ich nicht so gut aus dem Stegreif Fragen stellen kann; in was für einer Situation empfindest du dich? Findest du, daß ich dich mit meinen Fragen erdrücke oder zwinge?

11 So bemerkt auch Katrin Röggla zu den Voraussetzungen von Fichtes Fragen: „mit sicherheit basieren die gespräche auf wiederholtem fragen, vorfragen, zwischenfragen, die erst den fragekatalog entstehen ließen, der möglicherweise auch schriftlich vorlag“ (Röggla (2014), S.117).

12 Fichte (1983), S. 133.

13 Zum Begriff des ,kulturellen Kapitals‘ vgl. Bourdieu (1992), S. 49-80; Bourdieu (1982). 
Wolli: Ne, ich finde das ganz dufte, wenn man mal interviewt wird und was von sich reden kann und man selber in manchen Sachen klar denkt, man denkt dann über Dinge selbst nach oder über sich selbst, ich finde das ganz gut.

Fichte: Wir waren hier neulich zusammen und da hattet ihr ziemlich Trouble, Mauli und du. Hast du Lust über diesen Trouble zu reden? ${ }^{14}$

Bezugnahmen wie die Frage nach dem Befinden in der Interviewsituation und mehr noch die überraschende Frage nach Wollis letztem Beziehungsstreit suggerieren eine Vertrautheit zwischen den Interviewpartnern und erlauben es dem Fragenden, sich als Kenner, als Interessierten und aufmerksamen Beobachter, mitunter sogar als Insider zu positionieren. Sie markieren aber gleichzeitig eine bewusst vollzogene Grenzüberschreitung, die Fichte ungeachtet - oder gerade wegen - der Widerstände seines Gegenübers in aller Härte ausagiert.

Wolli: Ich kann dir noch nicht mal sagen, über was der Trouble war, das war irgend so ein ganz unbedeutender kleiner Käse.

Fichte: Ihr hattet euch Schimpfwörter an den Kopf geworfen.

Wolli: Ja, das machen wir manchmal, aber schon, wenn wir die Schimpfworte sagen, ist das Schlimmste vom Trouble vorbei, das ist so halb lustig gewesen.

Fichte: Wer hat denn angefangen mit den Schimpfwörtern?

Wolli: Ach, das kann ich nicht sagen, mal ich, mal sie.

Fichte: Was hast du ihr denn an den Kopf geworfen?

Wolli: Was weiß ich, irgend solchen Käse, ich weiß es nicht.

Fichte: Und das ging um die Studentin?

Wolli: Ja, um irgend so eine andere ging das, aber nicht speziell um die, ob das die Studentin war oder irgendeine andere.

Fichte: Ja, du bist neulich gesehen worden, in der Herbertstraße, mit der Studentin, die mit nacktem Arsch vor dir ging und du hautest ihr immer auf den Arsch mit deiner Hand.

Wolli: Das war in der Schmuckstraße, aber das haben nicht so viele gesehen, das war nachts. Nuja, so einen Blödsinn mache ich, da war ich auch betrunken dabei.

Fichte: Du bist aber gern mit ihr zusammen? ${ }^{15}$

\footnotetext{
14 Fichte (1983), S. 133.

15 Ebd., S. 133 f.
} 
Durch penetrantes Nachfragen fördert Fichte persönliche Details zutage, die in dem intimen Rahmen des undokumentierten Vieraugengesprächs geäußert, für den Portraitierten bezeichnend sind oder ihn mit den Widersprüchen seiner Selbstdarstellung konfrontieren. Nebensächlich erscheinende oder auch unangenehme Sachverhalte und Äußerungen werden aufgenommen und zum Thema gemacht. Gefragt sind die Routinen des Alltags, die alltäglichen und keineswegs aufregenden Erfahrungen, deren Erörterung ein konkretes und in all seinen Schattierungen gezeichnetes Portrait entstehen lässt. Denn so oberflächlich und unverfänglich das Gespräch über die ganz normalen Lebensumstände über weite Strecken erscheint, so unversehens und fast unbemerkt geht es Schritt für Schritt in intime Fragestellungen wie jene nach dem schwulen Begehren des Befragten über. Seitenlange Befragungen der Konsumgewohnheiten werden über die Frage nach der Konsumption von Sexualität bis hin zur Frage nach sexuellem Begehren und schwulen Alltagspraktiken ausgebaut:

Gibst du viel für Essen aus?/ Kaufst du im Delikatessengeschäft?/Was ißt du gerne?/Gibst du viel für dein Wohnen aus?/Du hast eine Reinemachefrau?/Gibst du viel für Kleidung aus?/Aber du ziehst dich gerne hübsch an./Du hast also Hosenanzüge aus sehr bunten Stoffen, ziehst du rosa Anzüge an.../Liebst du Parfüms?/Rauchst du viel?/Wieviel Zigaretten am Tag?/Aber trinken tust du nicht/...Was sammelst du für Bilder?/Wo kaufst du? ${ }^{16}$

$[\ldots]$

Und fürs Stoßen, legst du da viel an?/Ja, ist dir das nun nicht unangenehm zu bezahlen?/Machst du das regelmäßig?/Und was kostet dann so ein Abend?/Wenigstens./Und höchstens?/Auf den Strich bist du selbst nie gegangen? ${ }^{17}$

[...]

Hast du noch nie eine Mark dafür bekommen, daß du irgendwie mit einem Kerl was gemacht hast?/Weitergehende Sachen hast du nie mit Kerls gemacht?/Und du würdest das auch nicht gerne tun?/Das wäre aber die Bedingung, es muß eine Tante dabei sein?/Was machst du mit Mädchen?/Ja, und worauf stehst du am meisten?/Und du hast dir nie vorgestellt, was du mit einem Kerl machen würdest?/Aber du bist doch gelegentlich mal auf Klappen gegangen, was fandest du da geil, und was hast du da gemacht? ${ }^{18}$

$\begin{array}{ll}16 & \text { Ebd., S. 36-38. } \\ 17 & \text { Ebd., S. 46-48. } \\ 18 & \text { Ebd., S. 48-50. }\end{array}$


Wo die Kontinuitäten des ganz normalen Lebens erfragt und Gewohnheiten aufgelistet werden, sind also die außergewöhnlichen, die intimen und möglicherweise grenzüberschreitenden Fragestellungen nicht fern, wobei sich der diskontinuierliche Sprung, in den Fluss eines detaillierten Berichts eingebunden, kaum merklich vollzieht. Mit einfachen Auskünften jedenfalls gibt sich Fichte selten zufrieden. Durch stetiges Nachfragen, durch die Bitte um Wiederholung und Konkretisierung lässt er seine Collagen aus (un)sagbaren Geschichten entstehen.

Das gilt auch für eine hypothetische Form der Konkretisierung, die auf den ersten Blick im Gegensatz zu der eben beschriebenen Befragung der alltäglichen Umstände steht, tatsächlich aber lediglich eine Umstandsveränderung imaginiert, um wie im ersten Fall in kleinen Schritten eine Frage- oder Problemstellung zu vertiefen, sie spekulativ zu überdehnen und zuzuspitzen:

Du würdest also für Geld ziemlich viel tun/Wenn dir nun sehr viel Geld geboten würde, was würdest du noch tun?/Nun kommt aber ein ganz mieser Kerl und will ungeheure Perversitäten von dir. Würdest du die auch für Geld tun?/Wann würdest du glauben, daß du dich nicht mehr im Spiegel angucken kannst. ${ }^{19}[\ldots]$

Für eine Million würdest du was machen?/Also, einen Finger würdest du nicht für eine Million hergeben?/...Die Hand?/Die Hand nicht, wieviel Finger?/Einen für eine Million. ${ }^{20}$

Je unwahrscheinlicher, je absurder und spekulativer die Fragen, desto unumwundener kann der Befragte antworten. Selbst und gerade wenn er die Diskussion beenden, wenn er abbrechen und ausweichen will, hält diese Form des Fragens eine Option des Weitersprechens bereit. Realitätshaltige Aussagen erhält der Fragende, wenn wir diese Technik der ,spekulativen Konkretisierung' ernst nehmen, also gerade in jenem Moment, in dem nicht das Wirkliche, sondern das Mögliche, nicht das Wahrscheinliche, sondern das Unwahrscheinliche erörtert wird. Von der Last befreit, sich für seine Aussage oder deren Konsequenzen verantworten zu müssen, weil es sich ohnehin um Spekulationen handelt, kann der Befragte moralisch verwerfliche oder politisch zweifelhafte, normalerweise undenkbare und unsagbare Äußerungen tätigen. Seine Phantasie wird somit zum Realitätsanker, der den grundlegenden Subtext seiner Persönlichkeit zu erkunden hilft: das, was wir sein Wesen oder, noch präziser, seine Haltung nennen.

\footnotetext{
19 Ebd., S. $28 \mathrm{f}$.

20 Ebd., S. 149.
} 
So sehr sich Fichte dem Wesen seines Gegenübers annähert, indem er dessen Lebensumstände durchleuchtet, so konsequent erkundet er die Beschaffenheit seines eigenen Instrumentariums und die Kontexte der Befragung, indem er die Gesprächssituation in all ihrer Breite und Unvorhersehbarkeit zum Gegenstand des Textes macht. Wie sich im ersten der drei Gespräche zeigt, wird das rahmende Geschehen auch dort produktiv gemacht, wo es zuerst einmal nicht mehr als eine Unterbrechung war:

Fichte: Du, sag mal, in der Zwischenzeit, beim Band wechseln, hatten wir doch so einiges besprochen, ich finde das gar nicht so uninteressant. Wir hörten also noch mal die Frage von mir ab, und ich sagte, das hörte sich doch verdammt tuntig an, und da meintest du, das höre sich nicht tuntig an, du seist ja viel tuntiger im Sprechen als ich, das meine ich nicht, aber du sagtest, du seist nun eine volle Alte in deinen sexuellen Empfindungen, was meinst du damit?21

Mit diesen Worten setzt Fichte ein krisenhaftes Moment wie den Wechsel des tapes als ,epistemologischen Glücksfall ${ }^{22}$ ein, um die Kanäle und Medien der Kommunikation (also die Techniken der elektronischen Stimmreproduktion) und die libidinösen Subtexte des Interviewgesprächs (das tuntige Sprechen) offenzulegen und sie zum Anlass einer weitergehenden Befragung werden zu lassen. Er nutzt solche Ausfälle also auch, um die Begehrensstruktur seines Gesprächspartners zu erfragen oder aber, wie in folgendem Einschub, sein eigenes Frage-Begehren schonungslos auszustellen. Hier transkribiert Fichte den Inhalt eines Telefonanrufs, den Wolli während der Befragung entgegennimmt, kurzerhand in den Gesprächsverlauf hinein:

Fichte: Grüß ihn schön von mir.

Wolli: Viele Grüße soll ich sagen von Hubert - und ich soll dir sagen, ich hab ihm schön einen geblasen und er hat einen Bock drauf, vielleicht ist er doch schwul - jaja - da zieht er die Knie ein und rollt sich und legt den Kopf zurück - okay - Tschüß mein Guter - jaja, mach ich - alles okay Viele Grüße von Robert _23

\footnotetext{
21 Ebd., S. 119.

22 Joseph Vogl bezeichnet krisenhafte Szenarien als „epistemologische Glücksfälle“. Vgl. Vogl (2012), S. 68.

23 Fichte (1983), S. 144.
} 
Fichtes Entscheidung, die gesamte Situation, die Zwischenfälle und Unterbrechungen bis hin zu Füllwörtern und Lücken, das Stottern und Stocken, die Eingangssituation und das scheinbar nebensächliche Geplauder mit in das Gesprächsprotokoll aufzunehmen, erinnert an eine am Vorbild des „direct cinema“ orientierte Interviewtechnik, die der sogenannte „recording angel “24 Andy Warhol auf einem anderen Kontinent, jedoch etwa zu der gleichen Zeit wie Fichte für die 1969 gegründete Zeitschrift InterVIEW kultiviert und als „Stimmportrait“ bezeichnet. ${ }^{25}$ Während Warhol und Fichte absolut gegensätzlich an das Interview herangehen, was den Grad der Vorbereitung angeht, so teilen sie eine akribische Aufzeichnungspraxis, welche mit Grammatik, Syntax und Vokabular den Klassencharakter, die Dialekte und die Akzente des Sprechens ebenso in den Fokus rückt wie die störenden Faktoren innerhalb und außerhalb des Gesprächs, die Eingangs- und Ausgangssituationen und die medialen Eingriffe. Man kann sagen, dass Fichte mit der Transkription von Zwischensequenzen wie der eben zitierten Replik auf den Tonbandwechsel oder das Telefongespräch eine Kopie erstellt, die ihren Reproduktionscharakter so ernst nimmt, dass sie dessen Unsichtbarkeit und angebliche Neutralität parodiert und ad absurdum führt. Gleichzeitig weist Fichte mit der Publikation von Gesprächen, die sich im ,Off‘ einer offiziellen Interviewsituation entwickeln, präzise auf eine Begehrensstruktur von Fragendem und Befragtem hin, welche deren Motivationen ebenso regelt wie die Rollenverteilung zwischen ihnen.

Nun ist es naheliegend, die skizzierten Fragetechniken zur Verarbeitung des Impliziten - also der Lebensumstände und der Haltung des Befragten einerseits sowie der Kontexte, der Prä- und Subtexte des Interviewgesprächs andererseits - als Antwort auf dieselbe Frage zu verstehen: Wie lässt sich eine Geschichte von unten schreiben, ohne die großen Erzählungen zu reproduzieren, die das Wissen über den Befragten ebenso prägen wie dessen Selbstdarstellung? In demselben Maße nämlich, in dem diese Techniken gleichsam auf die Erkundung eines Unsagbaren zielen, lassen sie deutlich werden, dass eine Geschichte von unten zu schreiben für Fichte immer auch bedeutet, die Grenzen des Sagbaren auszutesten. Und das geht nur, indem das Fragen in

24 Theweleit (1994), S. 428.

25 Bob Colacello, der Chefredakteur der auf Filmkritiken und Celebrity-Interviews spezialisierten Zeitschrift, skizziert die Herangehensweise von Warhol folgendermaßen: „He told me that the best way to do an interview was to visit the subject, ideally in their home, with no questions and no preconceptions - with as empty a mind as possible. This way, the interviewer will get the most accurate and revealing image of the subject via the topics he or she chooses to discuss as well as the grammar, syntax and vocabulary used. If the tape is transcribed very accurately, with each ,uhm', err' and ,but' included, what is redacted is a voice portrait“ (Bockris (1979), S. 17). 
ein Nachfragen und das Sagen in ein Überdenken, ein Neu- und Anders-Sagen überführt wird. Nicht die Authentizität einer ursprünglichen Aussage lässt ein Bild der realen Person entstehen, sondern die unendliche Variation, welche die Aussage im Prozess der Befragung durchläuft. Durch die ständige Umgestaltung des Erkenntnisobjekts findet nämlich, wie Lenin in Materialismus und Empiriokritizismus bemerkt hatte, eine fortwährende Vertiefung der Erkenntnis des Realobjekts statt. ${ }^{26} \mathrm{Am}$ Ende des zweiten Interviews bringt der befragte Wolli diese Vorläufigkeit und Kommentierungsbedürftigkeit des entstandenen Portraits auf den Punkt, wenn er auf Fichtes Frage: „Glaubst du, daß es [das Interview] das Wesentliche deiner Person ausdrückt oder möchtest du noch etwas Wichtiges dazu sagen?“ antwortet: „Ne, das ist wohl schon so ziemlich alles, aber das ist natürlich nur in diesem Moment meine Person, weil man sich ständig ändert und wechselt, und gerade ich bin in den letzten Jahren im ziemlichen Wechsel begriffen, die letzten zwei Jahre.“27

Der Versuch, durch Eintragung der Umstände eine Variationslinie im Aussagen zu erzeugen, zieht sich bis in die Verarbeitung des Interviews hinein, wenn der Befragte - wie anfangs geschildert - noch einmal die Möglichkeit hat, zu seinen Aussagen Stellung zu nehmen. Statt das Interview mit dem Ende des Gesprächs für abgeschlossen zu erklären und die Deutungshoheit an den Autor Fichte abzugeben, übernimmt Wolli kommentierend dessen Bewertung. Kursivierungen mit Wollis Einlassungen durchziehen das gesamte Interviewprotokoll. Dass er mit Kommentaren wie „[m]ach ich ja einen kleinen Jesus aus dem Köhler!" ${ }^{\mu 28}$ oder "Geil. Später sage ich das Gegenteil!“29 nicht allein die Inkonsistenzen seiner eigenen Erzählung reflektiert, ist augenfällig; mit seinen reflexiven, oft jovialen oder ironischen Bemerkungen führt er - was viel weitreichender ist, - den Interviewleiter Fichte als einen keineswegs unangreifbaren Gesprächspartner vor, um die eigene Dominanz im Prozess der Befragung hervorzuheben. Denn sobald das Kriterium von Authentizität und Wahrhaftigkeit der Rede vom Befragten nicht ernst genommen und durch den Spaß an der Inszenierung ersetzt wird, sobald also die Erzählung nicht der Selbstoffenbarung, sondern der Selbstdarstellung des Befragten dient, muss der Interviewleiter sich den Zweifel am Erfolg eines auf authentische Aussagen abonnierten Interview-Projekts ebenso gefallen lassen wie die Frage, ob und inwieweit hier nicht der Befragte zum Regisseur oder Spielleiter geworden ist. Ganz demonstrativ behauptet Wolli seine Autorschaft, wenn er nachträglich in

\footnotetext{
$26 \quad$ Althusser 1972 (1968), S. 208.

27 Fichte (1983), S. 167.

28 Ebd., S. 96.

29 Ebd., S. 123.
} 
den Primärtext eingreift, ihn bewertet und bebildert - so etwa, wenn er seine damalige Aussage, Drogenkonsum verändere das Leben, mit den Erfahrungen der Folgejahre untermauert ${ }^{30}$ - oder auch qualitativ erweitert und modifiziert, beispielsweise mit der Ergänzung biographischer Narrative und Deutungsangebote. ${ }^{31}$ Wer in diesem Deutungskampf das letzte Wort hat, macht Wolli unmissverständlich klar, wenn er zur Erklärung seiner eigenen Aussagen intertextuelle Bezüge zu Fichtes Büchern herstellt, die er nicht nur gelesen hat, sondern auch zu erläutern und einzuordnen versteht. ${ }^{32}$

In Wolli Indienfahrer werden wir Zeuge eines Perspektiv- und Rollenwechsels, der sich im Prozess der Überarbeitung vollzieht: tritt der Befragte als Kommentierender auf, so begibt er sich in den Ring, um selbst zum Autor zu werden und um die Auslegung des Textes zu kämpfen, ihm seine Stimme erneut und einmal anders einzuschreiben, ihn zu gebrauchen und zu verwandeln. Das kommentierte Gesprächsprotokoll, das uns dieser Roman zu lesen gibt, bringt also nicht allein eine Reflexivitätssteigerung hervor, sondern eine Verlängerung der Gesprächssituation, ihre Übertragung und Erweiterung in einen schriftlichen Artikulationsraum, der hier nicht nur dem Autor Fichte, sondern auch dem Befragten selbst zur Verfügung steht, ihm erlaubt, zur Befragungssituation ebenso wie zu den getätigten Aussagen Stellung zu nehmen, sie noch einmal zu korrigieren, zu präzisieren oder zu bestätigen. Zusammenfassend heißt dies, dass durch Fichtes Fragetechnik des insistierenden Nachfragens einerseits und durch die fortlaufende Kommentierung des Interviewtextes (seiner Voraussetzungen, Umstände und Verarbeitungsformen) andererseits permanent Akzentverschiebungen erzeugt werden. Nicht nur die Autorität des Interviewleiters Fichte, sondern auch die autoritäre Setzung eines primären oder ursprünglichen Interviewtextes, der authentische Äußerungen mitzuteilen vorgibt, wird hiermit infrage gestellt. Im Gegensatz zu einem klassischen Kommentar, welcher der Autorität eines ursprünglichen Textes verpflichtet ist, erlaubt es ein kritischer Kommentar wie dieser, sich gegen die Autorität des Primärtextes zu wenden und ihn von den Kontexten seiner Entstehung und seines Gebrauchs her immer wieder neu zu bewerten. ${ }^{33}$

\footnotetext{
$30 \quad$ Ebd., S. 113 .

31 Ebd., S. $65 \mathrm{f}$.

32 Etwa in Bezug auf Fichtes Band Pubertät kommentiert Wolli: „Wenn ich das Buch ,Pubertät' nehme und die enormen Schwierigkeiten sehe - wäre der Pozzi nicht gekommen und hätte der Junge eine nette Braut gehabt, hätte er sich viele Sachen, Selbstquälereien erspart" (ebd., S. 106).

33 Zum kritischen Kommentar vgl. Benjamin (1991), S. 123-202; Brecht (1997), S. 513-530.
} 


\section{Der Proust-Fragebogen}

Der literarischen Verarbeitung von Fichtes Interviews mit Wolli geht eine Hörspiel-Vertonung voraus, die bereits 1972 im Rahmen seiner St. Pauli Interviews in Westdeutschem Rundfunk (WDR) und Norddeutschem Rundfunk (NDR) gesendet wird. Während es sich bei den Interviews mit Ulli, Gunda, Jonny und Sandra um originale Mitschnitte der im Jahre 1969 geführten Interviews handelt, so kommt dem zeitgleich entstandenen Interview mit Wolli in Anlage und Ausführung eine Sonderstellung in den St. Pauli Interviews zu. Vertont wurde nicht das gesamte Gespräch, sondern allein der sogenannte Proust-Fragebogen ${ }^{34}$, der am Ende des ersten Interviews steht. Um den ProustLiebhaber Wolli zu befragen, hatte Fichte ihm den Fragebogen eines Poesiealbums vorgelegt, den einst Proust beantwortet hatte:

Fichte: Wolli, du liebst Proust.

Wolli: Ich liebe Proust sehr.

Fichte: Als Proust vierzehn Jahre alt war, bat ihn Antoinette Faure eines Tages, auf den Fragebogen eines englischen Albums zu antworten. Ich möchte dir jetzt diese Fragen des englischen Albums stellen. ${ }^{35}$

Mit diesen Worten beginnt eine Befragung, die weniger authentisch ist, als sie sich zuerst einmal anhört. Denn es handelt sich bei dieser Aufzeichnung nicht einfach um die elektronische Reproduktion der ursprünglichen Befragungssituation, sondern um die nachträgliche Vertonung eines schriftlich fixierten Gesprächs. Dieses Interview ist „echt inszeniert“36 bzw. auf Echtheit hin inszeniert, stellt diese Inszenierungssituation aber in subtiler Weise aus; insbesondere Fichte versucht gar nicht ernsthaft, authentisch zu klingen wer darauf achtet, hört förmlich, dass er seine Worte vom Blatt abliest. Wir haben es insofern mit einem „Reenactment“37 im doppelten Sinne zu tun: Die

34 Literarisch verarbeitet findet sich der sogenannte Proust-Fragebogen in: Fichte (1983), S. 118-129.

35 Ebd., S. 118.

36 Hoffmann/Kaiser (2014).

37 Zu einer (Medien-)Philosophie des Reenactments vgl. Muhle (2013); Hal Foster hat im „Reenactment“ die zeitgenössische Form eines Realismus ausgemacht, der Wiederholung weder als Simulation noch - im Sinne eines "traumatic realism“ - als Annäherung an eine traumatische Vergangenheit interpretiert, sondern als quasi-experimentelles Unterfangen: „Lately, however, a further shift has occurred in fiction and art: repetition is not on the side of simulation, but neither does it circle around a traumatic past. Rather, its purpose is to produce an interruption, like a crack or a gap, that might allow a different reality to be glimpsed" (Foster (2018), S. 23). 
Wiederaufführung des Proust'schen Fragebogens durch Fichte und Wolli wird um eine Wiederaufführung des wiederaufgeführten Interviews ergänzt. Gleich mehrere Probleme stehen dabei zur Diskussion. Während Fichte in einem ersten Schritt die schriftliche Vorformatierung der mündlichen Fragen zum Thema macht, indem er von einem präskriptiven Modell der Befragung, nämlich dem Proust-Fragebogen ausgeht, bringt er durch die nachträgliche Inszenierung des Gesprächsprotokolls zweitens ein Unterscheidungsproblem zwischen Authentizität und Inszenierung auf den Punkt, das zweifachen Ursprungs ist: Zum einen resultiert dieses Problem aus dem Umstand, dass die Primärsituation von den Prozessen ihrer Be- und Verarbeitung kaum getrennt werden kann, weil sie immer nur durch letztere zugänglich ist; zum anderen daraus, dass die Inszenierung bereits in jenem Moment beginnt, in dem der Interviewte seine Gefühle und sein Wesen für die Aufnahme in Szene setzt, indem er also die Aufzeichnung als Bühne nutzt, um ,echte' Probleme aufzuführen.

Wenn also Fichte und Wolli gemeinsam ein Interview einlesen, das Fichte vorher aufgezeichnet und ins Protokoll übertragen hat und das bereits in seiner ursprünglichen Fassung nach dem Modell eines anderen Interviews also als Kopie - gefertigt war, so gewinnt diese Einlesung kommentierende Schärfe. Sie markiert jene Übertragungs- und Übersetzungsprozesse, die in jedem Interview unsichtbar mitprozessiert werden: die Verschaltungen von Mündlichkeit und Schriftlichkeit, von Authentizität und Inszenierung. Dies aber sind exakt jene Unterscheidungen, welche die Genese eines interviewbasierten Erkenntnisprozesses anleiten, dem Zeugen seine Autorität und der Aussage ihre Belastbarkeit verleihen. Äußerst komödiantisch wird mit den Mitteln der Inszenierung das Authentizitätsversprechen des Interviews infrage gestellt, das von einer eindeutigen Zurechnung von Sprecher- und Autorschaftspositionen ebenso abhängt wie von der programmatischen Ausblendung des Medien- und Schriftcharakters einer als unmittelbar imaginierten Mündlichkeit.

Betrachten wir die Befragung selbst, so stellt sich das Frage-Antwort-Spiel von Beginn an als Abfolge von Kommentierungen der Interview-Situation dar. Wolli beantwortet den Proust-Fragebogen, woraufhin Fichte einen ersten Kommentar einschleift, wenn er verliest, was Proust einst geantwortet hatte. Denn Wollis Antwort wird durch den von Fichte angestrengten Vergleich nachträglich zum Kommentar auf Proust und gleichsam zum Anlass einer weiteren, auf die Umstände der Befragung bezogen Stellungnahme:

Welche Eigenschaften schätzt du am meisten bei einem Mann?

Wolli: Den Mut, für das einzustehen, was er als richtig erkannt hat.

Fichte: Proust sagt:

Die Intelligenz, den Geist und den Sinn für das Moralische. 
Wolli: Ja, das finde ich ja sehr schön, aber ich darf bloß eins sagen, und der Proust sagt drei, vier Sachen, der macht ganze Aufzählungen.

Fichte: Du darfst ja auch drei, vier Sachen sagen.

Wolli: Nun, dann sag ich jetzt auch mehr, würde ich sagen. Ich bemühe mich hier, das alles in einem Satz immer zu fassen. ${ }^{38}$

Wolli benennt hier die Schematisierung des Interviews, also die dem Befragten durch Zeit- oder Platzmangel auferlegten Beschränkungen, die gewöhnlich durch das Befragungsmodell selbst oder durch dessen institutionelle Einbindung vorgegeben sind. Oder er rückt biographische Details des befragten Proust, seiner besonderen Situationen und Lebensumstände im Moment der Befragung als konstitutiven Bestandteil der getätigten Aussagen in den Blick:

Fichte: Also, welche Eigenschaften schätzt du am meisten bei einer Frau? Wolli: Den Mann, den sie liebt, glücklich zu machen.

Fichte: Proust sagt: Die Sanftmut, Natürlichkeit und Geist.

Wolli: Das ist es. Das ist so ähnlich.

Fichte: Was tust du am liebsten?

Wolli: Am liebsten mach ich Liebe, Haschisch rauchen und Musik hören. Fichte: Mehr nicht?

Wolli: Ich lese auch gerne, aber am liebsten mach ich Liebe, wenn du mich fragst.

Fichte: Proust antwortet: Lektüre, Träumerei, Verse, Geschichte und Theater.

Wolli: Ja, das ist auch schön, aber Proust war vierzehn Jahre, stell dir mal vor, der hätte gesagt, am liebsten mache ich die Liebe, da hätte man gesagt, was ist denn das für ein verkommenes Bürschchen, das konnte er ja nun schlecht sagen. ${ }^{39}$

Wolli wagt es also, die systematischen und historischen Umstände als konstitutive und prägende Momente der im Interview getätigten Aussage herauszustellen und die Antworten des großen Autors Proust damit gleichsam zu relativieren, statt sie als Ausdruck von dessen Wesen und Genialität zu verklären. Durch das Kommentar-Werden des Interviews maximiert sich diese Relativierung noch. In ein Kommentierungsgeschehen eingebunden, in dem jede Aussage als eine sekundäre und vorläufige markiert und damit zum Bestandteil

$38 \quad$ Fichte (1983), S. 118.

39 Ebd., S. $118 \mathrm{f}$. 
eines unendlichen Gesprächs geworden ist, geht es in diesem Interview nicht mehr primär um die Frage, wer hier den Ton angibt (letzteres wäre bei der Tonaufzeichnung von Interviews tatsächlich wörtlich zu nehmen), sondern vielmehr um die Frage, welche Akzentverschiebung die Aussage im Prozess ihrer Umschrift - von Proust über Fichte bis hin zu Wolli - erfährt. Das heißt, sobald der Interviewtext als eine Ineinanderschichtung von Kommentaren erscheint, als Dialogizität im emphatischen Sinne des Wortes, ${ }^{40}$ muss das Konzept der Autorschaft revidiert werden. Das anfangs benannte Versprechen des Interviews als eines Mediums sekundärer oder geteilter Autorschaft findet, so möchte ich damit behaupten, im Format des Kommentars seine Konkretisierung.

\section{Gespräch mit Wolli und Linda}

Eine weitere Reformulierung der Autorschaftsproblematik im Interview leistet Fichte in einem vierten und letzten im Dezember 1982 entstandenen Gespräch mit Wolli und Linda, das in dem Band Hamburger Hauptbahnhof. Register im Rahmen seiner Geschichte der Empfindlichkeit erscheint. ${ }^{41}$ Insofern der Befragte auf seine Aussagen aus Wolli Indienfahrer sowie auf die Konsequenzen der damaligen Veröffentlichung zu sprechen kommt, ist dieses Gespräch Fortsetzung, diskursive Überarbeitung und reflexives Einholen der früheren Interview-Aussagen. Gleichzeitig wird der Text von Fichte als ein Metakommentar über das Genre des Interviews und sein Verhältnis zur Dialogizität des Gesprächs entworfen. Anders als in den oben behandelten Fällen werden dabei nicht nur die ihm immanenten Motivationen und Vorannahmen, die situativen Rahmenbedingungen, die Medien und Verfahren der Verarbeitung, sondern darüber hinaus auch die textuellen Rahmenbedingungen zum Thema gemacht, die bei der Veröffentlichung zum konstitutiven Teil des Interviewtextes werden.

Wenn Fichte den Kampf um Autorschaft in einen dramatischen Plot ummünzt und diesen Plot als einen das Interview kommentierenden Rahmentext einsetzt, so bildet seine Setzung den Auftakt zu einem textimmanenten Kommentierungsgeschehen, das sich zwischen Haupt- und Neben-(=Para) texten entfaltet. So nimmt die narrative Eingangssequenz überraschenderweise den Status eines auktorialen Vorworts an, wenn sie parodistisch von der

\footnotetext{
40 Julia Kristeva hat Dialogizität in diesem Sinne unter Bezug auf Michail Bachtin mit dem Konzept der Intertextualität kurzgeschlossen und als einen unendlichen Kommentierungszusammenhang interpretiert. Vgl. Kristeva (1972), S. 345-375.

41 Fichte (1993), S. 51-181.
} 
schwierigen Anbahnung des folgenden Interviewgesprächs berichtet. ${ }^{42}$ Zum dramatisch gerahmten Binnentext wird mit dem Interview hingegen ausgerechnet jene Textsorte, der Gérard Genette die paratextuelle Funktion zugewiesen hatte, in einem öffentlichen Raum "anywhere out of the book ${ }^{443}$ zu zirkulieren. Der Paratext verliert mithin den Status des Beiläufigen und wird zum Haupttext, der die ihm eigenen Peritexte herausbildet.

Bei genauerer Durchsicht fällt auf, dass der gesamte Text von einem Spiel mit jenen „Paratexten“ geprägt ist, die Genette als jenes „Beiwerk des Buches“44 beschrieben hat, dem gleichwohl eine wesentliche Funktion zukommt: das Werk in der Öffentlichkeit zu „präsentieren“, seine Lektüre und seinen Konsum zu steuern. ${ }^{45} \mathrm{Zu}$ verstehen sind darunter mit Genette alle „Arten zusätzlicher, auto- oder allographer Signale, die den Text mit einer (variablen) Umgebung ausstatten und manchmal mit einem offiziellen oder offiziösen Kommentar versehen“:46 „Titel, Untertitel, Zwischentitel; Vorworte, Nachworte, Hinweise an den Leser, Einleitungen usw.; Marginalien, Fußnoten, Anmerkungen; Motti; Illustrationen; Waschzettel, Schleifen, Umschlag “47 etc. In Fichtes Gespräch mit Wolli und Linda sind dies zuerst einmal Titel und Datierung, die den Text als Dokument kennzeichnen und mit einer historischen Signatur versehen. Hatte Fichte seinen Band Wolli Indienfahrer mit dem Zusatz „roman“ versehen, wenngleich er aus einer Sammlung von Gesprächsprotokollen bestand, das Dokument also gleichsam mit der illokutorischen Anweisung versehen: „betrachten Sie bitte dieses Buch als Roman“,48 so findet hier eine umgekehrte Bewegung statt: Der Autor Fichte fordert uns auf, seinen Text als Dokument eines authentischen Geschehens zu lesen, obwohl er mit einer Fiktion eröffnet wird und mit einer theatralen Szene schließt; Regieanweisungen prägen das Ende dieses Textes.

Die Divergenz zwischen Authentizitätsanspruch und Inszenierungsverdacht erschwert im Gespräch mit Wolli und Linda nicht nur die Lektüre des

42 Jens Ruchatz' Analyse des „Interview[s] im Paratext“ geht auf die paratextuelle Funktion des Vorworts ein: „Insofern sie [die Vorworte] als rahmender Kommentar dazu ausersehen sind, die Lektüre von Interviews im Sinne der Interviewer, Herausgeber oder Verleger zu lenken, können die Interviewpublikationen beigegebenen Texte legitim als funktionales Äquivalent zu Paratexten fiktionaler Literatur analysiert werden“ (Ruchatz (2014a), S. 121-143, hier S. 121).

43 Genette (2001), S. 328.

44 Ebd., S. 10.

45 Ebd., S. 9.

46 Genette (1993), S. 11.

47 Ebd.

48 Genette (2001), S. 17. 
Interviews, das ja ein dokumentarisches Genre ist; sie prägt auch dessen Anbahnung, wie uns in der erwähnten Einleitungssequenz mitgeteilt wird. Der dramatische Plot der Rahmenhandlung ergibt sich nämlich aus dem Umstand, dass Fichtes Alter Ego Jäcki es ablehnt, ein Interview zu führen, bei dem die Dramaturgie bereits von Beginn an absehbar, durch den Befragten selbst geplant und vorgegeben, in dem das Authentische also nur Show ist.

Wolli fing eigentlich davon an.

Noch ein Interview und so weiter.

Das wäre dann das dritte.

Aber es fiel in eine Zeit, wo Jäcki überhaupt keine Lust hatte zu interviewen.

Und auch die News Außenseiter-Zuhälter verläßt St. Pauli mit 50 und geht nach Costa Rica aufs Altenteil ins Exil.

Ach!

News!

Jäcki zierte sich also.

Er wußte eh, was Wolli erzählen würde.

Jäcki wußte eh, wie es ablaufen würde. ${ }^{49}$

Jäckis Unlust, ein Interview zu führen, bei dem bereits von vornherein feststeht, was sein Interviewpartner ihm erzählen wird, speist sich jedoch, wie im Laufe des Gesprächs immer deutlicher wird, weniger aus dem Umstand, dass es hier um eine Inszenierung geht, sondern vielmehr daraus, wer in dieser Inszenierung Regie führt. Schwerer als der Mangel an Authentizität scheint eine für das Interview ungewöhnliche Verteilung von Redepositionen zu wiegen: Die Hierarchie zwischen Fragendem und Befragtem ist hier umkämpft, wenn nicht sogar verkehrt. Nicht der Interviewleiter hat hier die Hosen an, sondern der Interviewte selbst. So ist dann Wollis Versprechen, die Hose herunterzulassen, für den Autor Fichte in verschiedener Hinsicht attraktiv nicht allein als Authentizitäts- und Sensationsversprechen des Befragten, als Versprechen, sich zu entblößen und den Blicken des Gegenübers und der Öffentlichkeit auszusetzen, sondern auch als Versprechen auf die Erfüllung eines Frage-Begehrens, das Fichte alias Jäcki in zahlreichen Romanen als Motiv und Ausgangspunkt seiner Interview-Streifzüge inszeniert hat. Erst nachdem sich Wolli schließlich vor Jäcki auf die Knie wirft, ihn anfleht, nun endlich mit dem Interview zu beginnen und wieder und wieder beteuert "-Ich zieh auch die Hose runter./- Ganz bestimmt. Ich zieh die Hose runter./-Ich zieh die Hose ganz runter", beschließt Jäcki, dem zum Freund gewordenen Interviewpartner

Fichte (1993), S. 51. 
die Bitte nicht länger auszuschlagen: „Ist doch egal, ob ich ihn nun interviewe oder nicht. Wenn ihm so viel daran gelegen ist." ${ }^{50}$ Bis zum Ende bleibt jedoch unklar, wer die Dramaturgie des nun folgenden Interviews eigentlich verwaltet - der befragte Zuhälter Wolli oder der fragende Jäcki, der - zwischendurch einen Rollenwechsel zum Realautor Fichte vollziehend - das Interview eröffnet und zuletzt ja auch erzählerisch verarbeiten kann und muss. Zuletzt treten Wolli, Linda und der Interviewleiter gemeinsam als Schauspieler auf und von der Bühne ab:

Immer noch ein Zungenkuß des Normalen in den Schwulen rein. Linda in Reizunterwäsche oben weinend. 5. Stock. Jäcki, ich liebe dich.

Wolli im Pyjama, vollgepängt hinter Jäcki her. Dritter Stock. Jäcki, du bist mein Einzigster.

Linda im 4. Stock.

Jäcki, du bist mein Bruder.

Wolli, zweiter Stock.

Jäcki, ich liebe dich.

Linda:

Jäcki, ich liebe dich.

Wolli Erdgeschoß.

Mein Jäcki.

Costa Rica, dachte Jäcki.

Zungenkuß.

Minutenlicht.

Tür zu. ${ }^{51}$

Wenn sich also die erzählte Eingangsszene in das Dokument eines Gesprächs und das Dokument wiederum in eine von Regieanweisungen und dramatischem Dialog geprägte theatrale Schlussszene verkehrt wird, dann stellt sich schlussendlich weniger die Frage, wer diesen Text inszeniert, als vielmehr die Frage, wie er inszeniert wird.

Fichtes Spiel mit Paratexten geht diesem wie der Inszenierung auf der Ebene der literarischen Konstruktion nach. Die illokutionäre Funktion des Paratextes, der im Dienste des Haupt- oder Referenztextes steht, wird zum sichtbaren Eingriff, da seine Vervielfältigung konfligierende Aufforderungen produziert. In demselben Maße, in dem er eine Anweisung zur Lektüre des Referenztextes bereitstellt, besiegelt der Paratext, wie nun deutlich wird, den "Gattungsvertrag

$50 \quad$ Ebd., S. 52.

51 Ebd., S. 181. 
(oder -pakt) “52. Ziehen wir jedoch in Betracht, dass das von Genette vorgestellte Konzept des Paratextes, wie Natalie Binczek bemerkt, in Ermangelung eines anderen Konsistenzmerkmals letztlich an die Intention des Autors gebunden bleibt, ${ }^{53}$ so holt Fichte den Text, der ihm durch die Eigenmächtigkeit seines Gesprächspartners Wolli zu entgleiten drohte, durch den virtuosen Einsatz von Paratexten in die eigene Verfügungsgewalt zurück. Gleichzeitig zwingt die von Fichte demonstrierte Unmöglichkeit, die Kommentierungs- oder Verweiszusammenhänge zwischen Texten zu begrenzen (der eine Text wird zum Paratext des anderen), zur Einsicht in die Brüchigkeit dieses Vertrages: Wenn - um es zugespitzt zu sagen - ,jeder Kontext als Paratext wirkt“54, ist die Paratextualität, wie auch Genette am Ende seiner Einleitung erkennen muss, „vor allem eine Fundgrube von Fragen ohne Antworten“55.

\section{Nachwort zum Engagement}

Wenn Fichtes Interviews, wie eben geschildert, zum Schauplatz einer Befragung des Fragens - der Fragesituation, ihrer Bedingungen und ihrer Effekte werden, dann kommt das keineswegs dem einfachen Reflexivwerden eines methodischen Werkzeugs oder poetischen Verfahrens gleich, das Jens Ruchatz mit dem Begriff des sogenannten „Post-Interviews" ${ }^{\text {“56 }}$ adressiert hat. Fichtes Engagement, der eingreifende Charakter seiner Interview-Kritik verdankt sich nämlich nicht nur dem Aufgreifen und Offenlegen, sondern auch dem Überarbeiten jener Setzungen, die das Interview und dessen Verarbeitung prägen.

52 Genette (2001), S. 12.

53 Natalie Binczek hat diese Bindung der Rahmentexte an die Werkkategorie und damit letztlich an die Autorinstanz mit Blick auf Gérard Genettes Paratextkonzept problematisiert: „An dem Punkt jedoch, an dem er die buchbedingte Einheit (des Peritextes) verlässt, um in öffentliche Bereiche (des Epitextes) auszuschwärmen, die sich in anderen medialen Formen ereignen und wie Buchbesprechungen in der Zeitung etwa, Interviews im Fernsehen oder private Korrespondenzen in Briefen das, was als Referenztext vorausgesetzt werden muss, allenfalls als thematischen Bezug aufrechterhalten, greift Genette auf die Instanz des Autors zurück und verlegt das Paratextkonzept auf die Werkkategorie. So führt er zuerst das Buch gegen das Werk an, um schließlich dieses mit Hilfe der auktorialen Kategorie doch zu rehabilitieren“ (Binczek (2004), S. 118).

54 Genette (2001), S. 15.

55 Ebd., S. 13 .

56 Jens Ruchatz bezeichnet als „Post-Interviews“ jene „Interviews, die, indem sie Interviews sind, die Strukturen und Bedingungen des Interviews thematisieren“ (Ruchatz (2014b), S. 47). Vgl. auch Ruchatz (2014a), S. 321-333. 
Was Fichtes Interviews zur Interview-Kritik werden lässt, ist der Umstand, dass sie einen Autoritätsverlust evozieren, der all jene Setzungen der InterviewEpisteme betrifft, die einer im Frage-Antwortspiel erzeugten Aussage ihre Geltung verleihen. Die Unterscheidung von primärem und sekundärem Text (Wolli Indienfahrer) ist davon ebenso betroffen wie jene von authentischem und inszeniertem Ausdruck (Proust-Fragebogen) oder aber von Monologizität und Dialogizität (Gespräch mit Wolli und Linda). Mit letzterem steht auch die grundlegende Unterscheidung zwischen Interview und Gespräch, zwischen hierarchischer und freundschaftlicher Kommunikation, Verhören und Vernehmen auf dem Spiel. Wenngleich diese Unterscheidung in Fichtes Interview-Literatur fortwährend aufgerufen wird - am explizitesten wohl mit der Benennung zweier Urszenen: „König Ödipus“ und das „Gastmahl“ ${ }^{457}$-, verliert sie in jenem Moment an Bedeutung, in dem das Interview durch insistierendes Nachfragen einerseits und die fortwährende Überarbeitung des Interviewtextes andererseits in einen unendlichen Kommentierungs- oder Gesprächszusammenhang übergeht. ${ }^{58}$ Engagiertes Fragen wird bei Fichte in diesem Sinne weder im Sinne einer reflexiven Haltung, noch als Protest- oder Gegenprogramm entworfen, sondern als Arbeit an jenen Setzungen institutioneller, methodischer und ästhetischer Art, welche die Dramaturgie von Frage und Antwort im Interview strukturieren. Engagierte Kunst heißt hier, um Adornos Idee des Engagements aufzunehmen, „nicht: Alternativen pointieren, sondern, durch nichts anderes als ihre Gestalt, dem Weltlauf widerstehen, der

57 „Das Feature (die zwanghafte Ungebundenheit) und das Interview (Beichte, Verhör, Psychoanalyse, Gespräch) - König Ödipus und Das Gastmahl“ - mit diesen Worten bringt Fichte die zweifache Verpflichtung seiner Interviewliteratur auf den Punkt. König Ödipus, der seine Frau Iokaste, den Seher Teiresias sowie einige Boten als Zeugen befragt, um Gewissheit über seine eigene Herkunft zu erhalten, wird bei Fichte zur Urszene eines unabschließbaren, auf gnadenlose Selbstbefragung, -zerstückelung und -erkundung ausgehenden aufklärerischen Frage-Begehrens, während mit dem Gastmahl als zweiter Urszene ein auf Erkenntnis ausgerichteter dramatischer [?] Dialog benannt und das freundschaftliche Gespräch als ,Symposium' der Formen reinterpretiert wird. Sein deutsches Lesebuch sei ein „Feature aus Gedichten, Theaterstücken, Features, Prosa - ein Lesebuch als ,Symposium “', so formuliert er einmal (Fichte (1987a), S. 21).

58 Seine Bewunderung für die modernen Schriftsteller wie auch die Modernität von Herodot hatte Fichte damit begründet, dass sie Kommentar und Bericht auf radikale Weise zu trennen wussten, ein journalistisches und poetisches Verfahren miteinander kombinierten: „Herodot trennt auf eine moderne Weise Bericht und Kommentar, zu einem Vorfall zitiert er oft mehrere Zeugenaussagen und überläßt dem Leser das Fazit./Es ist ein journalistisches Verfahren und ein poetisches“" (Fichte (1987b), S. 383). 


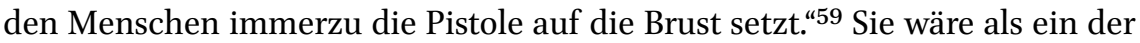
Kategorisierung entgegengesetztes Formexperiment, als Arbeit an den Grundlagen und der Formgebung des Interviews selbst zu verstehen.

\section{Literatur}

Adorno, Theodor W. (1973): „Engagement“, in ders.: Zur Dialektik des Engagements. Aufsätze zur Literatur des 20. Jahrhunderts II. Frankfurt am Main: Suhrkamp, S. 7-31. Althusser, Louis, Der Gegenstand des Kapital, in ders./Balibar, Etienne, Das Kapital lesen. Bd. 1, Hamburg: Reinbek 1972 (1968).

Benjamin, Walter (1991): Goethes Wahlverwandtschaften, in ders.: Gesammelte Schriften. Band I.1. Hg. von Rolf Tiedemann und HermannSchweppenhäuser, Hermann. Frankfurt am Main: Suhrkamp, S. 123-202.

Bies, Michael (2014): „Ethnographie des Romans. Hubert Fichtes Forschungsbericht“, in: Kammer, Stephan/Krauthausen, Karin (Hg.): Hubert Fichtes Medien. Berlin, Zürich: Diaphanes, S. 147-163.

Binczek, Natalie (2004): „Epistolare Paratexte. Über die ästhetische Erziehung des Menschengeschlechts in einer Reihe von Briefen“, in: Kreimeier, Klaus/Stanitzek, Georg (Hg.): Paratexte in Literatur, Film, Fernsehen. Berlin: De Gruyter, S. 117-135.

Bockris, Victor (1979): „Andy Warhol the writer“, in: McCabe, Colin/Francis, Mark/ Wollen, Peter (Hg.): Who is Andy Warhol? London: British Film Institute, S. 17-21.

Böhme, Hartmut (1992a): Hubert Fichte. Riten des Autors und Leben der Literatur. Stuttgart: Metzlersche Verlagsbuchhandlung, S. 36-42.

Böhme, Hartmut (1992b): „Die anthropologische und autobiographische Dimension der Frage und des Fraglichen im Werk Hubert Fichtes", in: Barkhoff, Jürgen/Sagarra, Eda (Hg.): Anthropologie und Literatur um 180o. München: Iudicium Verlag, S. $247-275$.

Bourdieu, Pierre (1982): Die feinen Unterschiede. Kritik der gesellschaftlichen Urteilskraft. Frankfurt am Main: Suhrkamp.

Bourdieu, Pierre (1992): „Ökonomisches Kapital - Kulturelles Kapital - Soziales Kapital", in ders.: Die verborgenen Mechanismen der Macht. Hamburg: vsA Verlag, S. $49-80$.

Brecht, Bertolt (1997): „Der Fatzerkommentar“, in ders.: Große kommentierte Berliner und Frankfurter Ausgabe. Band 10.1. Stücke 10. hg. von Hecht, Werner/Knopf, Jan/

59 Adorno (1973), S. 11. Adorno formuliert seine Skizze engagierter Kunst in Abgrenzung von Jean Paul Sartres Entwurf engagierter Literatur als eines "Appells“ an die Freiheit des Lesers, „daß sie an der Produktion seines Werks mitarbeite“. Vgl. Sartre (1981), S. 41. 
Mittenzwei, Werner/Müller, Klaus-Detlef. Berlin, Weimar, Frankfurt a. M.: Aufbau/ Suhrkamp, S. 513-530.

Brecht, Bertolt (1972): Interviews aus dem Palais d'Amour etc. Reinbek: Rowohlt.

Brecht, Bertolt (1989): Forschungsbericht. Roman, in ders.: Die Geschichte der Empfindlichkeit. Band Xv, hg. von Lindemann, Gisela in Zusammenarbeit mit Mau, Leonore. Frankfurt a. M.: Fischer.

Brecht, Bertolt (1983): Wolli Indienfahrer. Roman. Frankfurt am Main: Fischer.

Brecht, Bertolt (1987a): Elf Übertreibungen. Einführung in ein Lesebuch. Dakar, Juni 1976, in ders.: Geschichte der Empfindlichkeit. Homosexualität und Literatur 1. Polemiken. Hg. von Lindemann, Gisela in Zusammenarbeit mit Mau, Leonore. Frankfurt a. M.: Fischer, S. 9-23.

Brecht, Bertolt (1987b): Mein Freund Herodot, in ders.: Geschichte der Empfindlichkeit. Homosexualität und Literatur 1. Polemiken. Hg. von Lindemann, Gisela in Zusammenarbeit mit Mau, Leonore. Frankfurt a. M.: Fischer, S. 381-407.

Brecht, Bertolt (1993): Gespräch mit Wolli und Linda. 12. Dezember 1982, in ders.: Geschichte der Empfindlichkeit. Hamburger Hauptbahnhof. Band XIX. Register. Hg. von Lindemann, Gisela in Zusammenarbeit mit Mau, Leonore. Frankfurt a. M.: Fischer, S. 51-181.

Foster, Hal (2018), „Real Fictions“, in: Thanner, Veronika/Vogl, Joseph/Walzer, Dorothea (Hg.): Die Wirklichkeit des Realismus. Paderborn: Fink, S. 17-27.

Genette, Gérard (1993): Palimpseste. Die Literatur auf zweiter Stufe. Frankfurt a. M.: Suhrkamp.

Genette, Gérard (2001): Paratexte. Das Buch vom Beiwerk des Buches. Frankfurt a. M.: Suhrkamp.

Hoffmann, Torsten/Kaiser, Gerhard (Hg.) (2014): Echt inszeniert. Interviews in Literatur und Literaturbetrieb. Paderborn: Fink.

Kristeva, Julia (1972): „Bachtin, das Wort, der Dialog und der Roman“, in: Ihwe, Jens (Hg.): Literaturwissenschaft und Linguistik. Ergebnisse und Perspektiven. Band 3: Zur linguistischen Basis der Literaturwissenschaft II. Frankfurt a. M.: Athenäum, S. $345^{-} 375$.

Linck, Dirck (1995): „Nun ist alles anders“ Über Hubert Fichtes Reise-Begehren“, in: Böhme, Hartmut/Tiling, Nikolaus (Hg.): Medium und Maske: die Literatur Hubert Fichtes zwischen den Kulturen. Stuttgart: M \& P Verlag für Wissenschaft und Forschung, S. 23-54.

Muhle, Maria (2013): „History will repeat itself. Für eine (Medien-)philosophie des Reenactment“, in: Engell, Lorenz/Hartmann, Frank/Voss, Christiane (Hg.): Körper des Denkens. Neue Positionen der Medienphilosophie. München: Wilhelm Fink, S. $113-135$.

Röggla, Kathrin (2014): „der akustische fichte“, in: Kammer, Stephan/Krauthausen, Karin (Hg.): Hubert Fichtes Medien. Zürich,Berlin: Diaphanes, S. 111-125. 
Ruchatz, Jens (2014a): Die Individualität der Celebrity. Eine Mediengeschichte des Interviews. Konstanz, München: UvK Verlagsgesellschaft.

Ruchatz, Jens (2014b): „Interview-Authentizität für die literarische Celebrity. Das $\mathrm{Au}-$ toreninterview in der Gattungsgeschichte des Interviews“, in: Hoffmann, Torsten/ Kaiser, Gerhard (Hg.): Echt inszeniert. Interviews in Literatur und Literaturbetrieb. Paderborn: Wilhelm Fink, S. 45-63.

Sartre, Jean-Paul (1981): Was ist Literatur?, in ders.: Gesammelte Werke in Einzelausgaben. Schriften zur Literatur. Band 3. Hg. von Traugott König. Reinbek: Rowohlt.

Seifert-Waibel, Miriam (2005): Ein Bild, aus tausend widersprüchlichen Fitzeln. Die Rolle der Collage in Hubert Fichtes Explosion und Das Haus der Mina in São Luiz de Maranhão. Bielefeld: Aisthesis Verlag.

Theweleit, Klaus (1994): Buch der Könige. Band 2y: Recording angels' mysteries. Basel, Frankfurt a. M.: Stroemfeld/Roter Stern.

Vogl, Joseph (2007): „Kluges Fragen“, in: Gruber, Klemens/Schulte, Christian (Hg.): Maske und Kothurn. Die Bauweise von Paradiesen. Wien, Köln, Weimar: Böhlau, S. 119-129.

Vogl, Joseph (2012): „Gezähmte Zeit. Finanzialisierungsprozesse und ihre Medien“, in: Berz, Peter/Kubaczek, Marianne/Laquièze-Waniek, Eva/Unterholzner, David (Hg.): Spielregeln. 25 Aufstellungen. Eine Festschrift für Wolfgang Pircher. Zürich: Diaphanes, S. 61-71.

Weber, Samuel M. (1975): „Der Einschnitt. Zur Aktualität Vološinovs“, in: Vološinov, Valentin N.: Marxismus und Sprachphilosophie. Grundlegende Probleme der soziologischen Methode in der Sprachwissenschaft. Hg. von Samuel M. Weber. Frankfurt a. M., Berlin, Wien: Ullstein, S. 9-47. 